\title{
Protée
}

\section{La littérature à l'écran. Approches et limites théoriques}

\section{Alexie Tcheuyap}

Volume 29, numéro 3, 2001

Iconoclasmes : langue, arts, médias

URI : https://id.erudit.org/iderudit/030640ar

DOI : https://doi.org/10.7202/030640ar

Aller au sommaire du numéro

Éditeur(s)

Département des arts et lettres - Université du Québec à Chicoutimi

ISSN

0300-3523 (imprimé)

1708-2307 (numérique)

Découvrir la revue

Citer cet article

Tcheuyap, A. (2001). La littérature à l'écran. Approches et limites théoriques. Protée, 29(3), 87-96. https://doi.org/10.7202/030640ar

\section{Résumé de l'article}

Du littéraire au filmique, quel est le lieu de la signification ? Qu'est-ce qui légitime la différence du texte dérivé ? Est-ce le code sémiotique, l'idéologie, l'institution ou la répétition poétique ? Voilà quelques-unes des interrogations que soulève cet article. D’André Bazin à André Gaudreault, la sémiotique du cinéma semble opérer de simples mutations de pouvoirs créateurs en passant de la prééminence du texte littéraire à celle de la machinerie audiovisuelle. Il importe pourtant, vu les répétitions poétiques émaillant l'histoire culturelle universelle, de recentrer le débat de la différence non sur un code, mais sur la fréquence de l'acte créateur. Une telle perspective de réécriture resitue le débat théorique dans une perspective transgénérique, transcodique et translinguistique. 


\title{
LA LITTÉRATURE À L'ÉCRAN APPROCHES ET LIMITES THÉORIQUES
}

\author{
AleXIE TCHEUYAP
}

La reprise filmique des textes littéraires soulève un certain nombre de questions qui demeurent sans réponse. Il s'agit principalement de la capacité des images industrielles à soutenir une création qui, tout en partant d'un rapport de duplication, parvienne, au terme de la réalisation, à évacuer quelques doutes. Le principal porte sur la prééminence plus ou moins implicite de la source littéraire sur la version filmique. L'Avant-garde française a d'abord considéré le phénomène comme un pis-aller honteux, avant qu'André Bazin tente de remettre les choses à leur place: la plupart des grands chefs-d'œuvre du cinéma contemporain sont dérivés de textes littéraires. Son plaidoyer formulé «[p] our un cinéma impur» constitue à cet égard un point théorique décisif sur la nature des rapports entre la littérature et le septième art condamné à «l'impureté». Il écrit notamment:

Sachons donc d'abord voir ce que les meilleurs films contemporains doivent aux romanciers modernes [...] Alors, loin de nous scandaliser des adaptations, nous y verrons, sinon hélas un gage certain, du moins un facteur possible de progrès du cinéma. Tel qu'en lui-même, le romancier le change! ${ }^{1}$

Largement exposées dans son essai, les hypothèses de Bazin n'ont pas seulement enrichi le débat sur la reprise filmique des textes littéraires, elles l'ont aussi miné. La prééminence du texte et de la source littéraires impose en effet un certain tribut et même une servitude au réalisateur dont l'œuvre n'est pas considérée dans son intégrité ou son indépendance, mais se trouve réduite à sa capacité de mémoire et de reflet. En d'autres termes, l'un devient le lieu, le centre de gravité, la norme à partir de laquelle est évaluée l'autre. En dépit de l'antériorité d'une démarche largement critiquée, cette étude s'attachera à retraverser la démarche de Bazin pour montrer que son point focal subsiste et vient de resurgir sous une forme voilée.

En effet, opérant les critiques les plus récentes des paradigmes baziniens, les théoriciens de la transécriture ${ }^{2}$ inversent le procès épistémologique et, comme nombre de leurs prédécesseurs, soufflent à la littérature un pouvoir qui est aussitôt conféré au cinéma: on demeure, dans la lignée bazinienne, figé dans l'idéologie du code sémiotique, du média. Le problème demeure ainsi irrésolu par ce renversement du schéma évaluateur. Il légitime en effet la différence du texte filmique par la nature du nouveau média qui imposerait une nouvelle "fidélité». La question, fondamentale, de la poétique et de la signification se trouve prise entre deux pôles institutionnels: la préséance du texte littéraire et une sorte de déterminisme technologique. Dans un tel contexte, fondant la différence sur la répétition, l'hypothèse de la réécriture, dont le procès théorique est mis en ouvre dans cet article, déplace le champ de la réflexion par l'élaboration d'une poétique qui se libère définitivement de l'héritage bazinien pour recentrer la démarche théorique sur les enjeux de la création et de la poétique, véritable paramètre "intermédial» pouvant s'émanciper du pouvoir des mots.

\section{L'héritage bazinien: du pouvoir des mots...}

André Bazin a très utilement jeté les bases d'une étude comparative entre le cinéma et la littérature. Il a princi- 
palement démontré qu'il ne saurait exister un quelconque cloisonnement entre les arts, lesquels s'impliquent fatalement les uns les autres. Mais il a aussi, à la lettre, sabordé cette démarche comparative en léguant à la critique deux termes qui semblent avoir la peau dure: fidélité et trahison. Le réalisateur qui, au lieu de construire un scénario «original», le fonde sur un texte littéraire, ne voit plus, de ce fait, son œuvre considérée dans son identité. Un certain fétichisme impose le fantôme du texte-source dont on se sert pour justifier une improbable recherche des diverses structures dans le texte dit d'arrivée. Bazin est ferme, le cinéaste ne saurait s'affranchir du paramètre de la fidélité. Elle est, pour lui, rien de moins qu'une sorte de «servitude» ${ }^{3}$. Bien plus:

On peut poser que, dans le domaine du langage et du style, la création cinématographique est directement proportionnelle à la fidélité. Pour les mêmes raisons qui font que la traduction mot à mot ne vaut rien, que la traduction trop libre nous paraît condamnable, la bonne adaptation doit parvenir à restituer l'essentiel de la lettre et de l'esprit. 4

Ces deux derniers termes constituent les autres versants de l'héritage bazinien, repris par maints critiques. Bien que flous, et à la limite, intuitifs, ces critères sont pris en compte par Alain Garcia dont le livre ne va pas sans contradictions et ambiguïtés. Selon lui, l'œuvre originale demeure le texte de référence auquel le cinéaste doit se référer. Dans ce cas, le Journal d'un curé de campagne de Robert Bresson en est un exemple type puisque la quête de l'origine a la primauté sur la création et l'inventivité. Tout est question de degré, le cinéaste ne peut se donner les libertés qu'il veut:

[...] le scénariste adaptateur n'a pas le "droit» de faire ce que bon lui semble du chef-d'ouvre qu'il transpose à l'écran [...] Respect à la lettre ou à l'esprit, l'une de ces deux situations est suffisante; et si la trahison des deux ne nous intéresse pas du tout, c'est bien entendu le respect des deux, le respect de l'auteur et de son sujet qui forcent notre admiration et font du film, pour nous, un chef-d'euvre d'adaptation. 5

Cette quête imaginaire de la fidélité, de l'esprit ou de la lettre, est aussi le fait de Gene D. Philips. Dans son livre, Hemingway and Film, il pose que le film doit restituer les thèmes et les intentions originelles de l'auteur. Il est plus formel:
The faithful film adaptation must preserve the spirit and thrust of the original work, regardless of superficial liberties taken with the plot and dialogue of the parent work in retooling it for the screen. ${ }^{6}$

Dans un article pourtant destiné à montrer les limites d'une critique fondée sur le critère de fidélité, Christopher Orr écrit dès le premier paragraphe, sur un mode tout aussi prescriptif, voire radical: "A good adaptation must be faithful to the spirit of its literary source" ${ }^{7}$. Qu'est-ce donc que la lettre? Et l'esprit? Ne s'agit-il pas de critères rigoureusement subjectifs, surtout textuels, basés sur la capacité du spectateur à se souvenir du texte littéraire d'où a été tiré le film? L'enjeu de l'emprunt est-il de resituer ou de restituer? La seconde démarche est-elle possible?

Il est fondamental de relever que celui qui prend l'initiative de porter un texte littéraire à l'écran a moins en vue de rendre justice à une œuvre que de faire un bon film à partir d'une lecture personnelle ou encore des goûts d'un public. C'est entre autres pourquoi le film Les Misérables de Frank Lloyd (1918) est différent de celui, fait en trois parties, de Raymond Bernard (1933), ou de celui de Claude Lelouch, par lequel le roman de Victor Hugo devient Les Misérables du XXe siècle (1995). De plus, il est rigoureusement impossible de rendre à l'écran la lettre ou même l'esprit d'un texte littéraire, ce qui impliquerait un concept de "totalité» incompatible avec tout texte. Celui-ci se trouve simplement engagé, du fait de la reprise, dans un processus herméneutique. Pareille entreprise est utopique, vouée à l'échec et, de plus, matériellement impossible.

La fidélité à l'esprit constitue un autre versant de ce mythe, car la notion suppose, ainsi que le suggère Phillips, qu'il existe un sens et un projet de l'auteur, ce qui semble douteux. Le film, texte dérivé et autonome, devient ainsi captif d'un autre, dit "original», que l'utopie critique soustrait à jamais à l'appréhension ou, du moins, au concret travail des significations, en le reléguant à la sphère indéterminée de l'abstraction qui trouve ainsi un terme commode par son imprécision et son ambiguïté: «l'esprit». Devant cette situation sans issue, d'autres théoriciens ont procédé par étapes. L'absolu de la fidélité étant trop incertain, ils ont élaboré d'autres paradigmes tout aussi problématiques, qu'il demeure délicat de délimiter avec rigueur.

On retrouve encore Alain Garcia, lequel structure son livre en trois parties: l'adaptation qui «trahit le cinéma en 
étant trop près de la littérature», l'adaptation libre qui «trahit le roman en prenant trop de distance vis-à-vis du roman", puis la transposition qui, elle, «ne trahit ni l'un ni l'autre en se situant aux confins de ces deux formes d'expressions artistiques» ${ }^{8}$. Dans le même registre théorique, François Baby considère que

[...] l'adaptation stricte est caractérisée par un haut niveau de fidélité par rapport à l'auvre originale. On ne retrouvera dans le produit final que les modifications imposées par le changement de médium et par l'observance des contraintes que ce changement entraîne.

Vient ensuite l'adaptation libre, caractérisée par «un degré moindre de fidélité à l'œuvre originale» et qui ne doit pas «modifier fondamentalement le sens et la portée de l'œuvre originale». L'adaptation dite "d'après» est la moins «fidèle»: [elle est] caractérisée par un faible niveau de fidélité par rapport à l'auvre originale. En effet, l'auteur s'inspire plus ou moins directement de l'ceuvre d'origine, la plupart du temps d'ailleurs, surtout au niveau de l'armature. Elle entraîne donc généralement un travail important de création de la part de son auteur. ${ }^{10}$

On se situe dans le même réseau théorique que Garcia, où la liberté du créateur est paradoxalement rivée à ses degrés de fidélité au texte littéraire. Une incertitude encombre les frontières variables entre les divers degrés d'évaluation du film dérivé de la littérature. À ces catégories, il faut ajouter celles de Geoffrey Wagner. D'abord, ce qu'il nomme transposition, «in which a novel is given directly to the screen with a minimum of apparent interference» ${ }^{11}$. Par le commentaire,

[...] an original is taken and either purposely or inadvertly altered in some respect... when there has been a different intention on the part of the film maker, rather than infidelity or outright violation. 12

Enfin, l'analogie «which must represent a fairly considerable departure for the sake of making another work of art» 13 .

À partir des exemples précédents, on voit que les reprises cinématographiques des textes littéraires ont largement été abordées par la critique en termes de pouvoir: le mot assujettit les images, la littérature fait écran au cinéma. D'autre part, il se révèle un brouillage, car même ceux qui adoptent des concepts semblables n'y mettent pas forcé- ment le même contenu. On le voit notamment avec le cas de la transposition et de l'adaptation libre qui ne véhiculent pas les mêmes opérations chez Garcia, Baby ou Wagner. Tout cela témoigne d'un certain malaise épistémologique. Certes le consensus n'est guère fréquent dans le domaine de la science et ce n'est pas forcément un critère. Mais il n'en demeure pas moins vrai que les bases théoriques des propositions précédentes vacillent. Elles sont trop arbitraires, trop floues. Qui dira exactement, avec le maximum de précision, en quoi consiste «l'esprit» ou «la lettre» d'une œuvre littéraire ou filmique? Comment trancher quant au degré, supérieur ou moindre, de fidélité du film de Mara Mattuschka (1987) qui, comme bien d'autres, est basé sur Les Misérables? Cette incertitude théorique, identifiable dès Bazin, voudrait ramener à l'altérité de la version filmique l'identité improbable du texte littéraire. Pour MarieClaire Ropars-Wuillemiers, une telle approche

[...] est en réalité une hiérarchie, entièrement réglée par le principe d'identité: c'est en fonction de l'ouvre originelle qu'on évalue l'intervention du cinéma; et c'est la fidélité ou la force de la transposition, l'aptitude à traduire ou à relancer un chef-d'cuvre supposé rester intact qui vont déterminer la qualité d'un film issu d'un texte. Se dévoile alors une contradiction implicite entre un plaidoyer pour l'impureté cinématographique, qui ferait du cinéma un opérateur critique de la question de spécificité, et la reconduction d'une échelle de valeurs référée à la littérature, qui pourrait seule opérer la purification nécessaire du septième art. ${ }^{14}$

Dans Novels into Films, George Bluestone soutient que les jugements de fidélité supposent que l'intrigue et les personnages romanesques soient automatiquement transférables et substituables, que le roman est la norme dont le film s'éloigne à ses risques et périls, que les "déviations" ne sont permises que pour des raisons certes imprécises, mais réglées par la distance prise de l'original. Il est important, redisons-le, de désacraliser le texte-source et de prendre enfin le texte dit d'arrivée pour ce qu'il est et ce qu'il est seulement: un autre texte, c'est-à-dire un texte nécessairement autre. Car en passant du littéraire au filmique, les changements sont inévitables:

[...] mutations are probable the moment one goes from a given set of fluid, but relatively homogeneous, conventions to another; $[. .$.$] changes are inevitable the moment one abandons$ the linguistic for the visual medium. ${ }^{15}$ 
Il s'agit dès lors de chercher un autre versant de la théorie: il pose le changement comme inévitable et même comme une nécessité intrinsèque, constitutive de l'œuvre. La création procède par mutation de code et déplacement sémiotique. C'est le règne de l'image et de ses pouvoirs.

2. ... au pouvoir de l'image: le déplacement sémiotique

La base de la théorie que nous désignons par «déplacement sémiotique» est le changement de code. La littérature et le cinéma constituant deux langages différents, l'emprunt impose inévitablement des transformations. Les tenants du déplacement sémiotique désignent presque tous l'opération de duplication par un terme au préfixe seyant: trans-. La logique est donc celle du mouvement, c'est-àdire de l'instabilité, de la mobilité. Dans une telle perspective, la version filmique du texte littéraire est un dérivé qu'il est difficile de figer par une formule ou par quelque règle. Paradoxalement, l'un des fondements implicites du déplacement sémiotique c'est l'étanchéité des deux systèmes de signification - la littérature et le cinéma. Pour Linda Coreman, le cinéma se charge de mettre en place ses propres réseaux sémantiques et peut, éventuellement, actualiser des sèmes dispersés dans le texte littéraire. Ainsi, la spécificité du Hamlet de Celestino Coronado (1976) est légitimée par l'emploi de langages audiovisuels. La différence avec le texte de départ n'est ni un accident, ni une tare, car l'œuvre littéraire portée à l'écran est forcément différente, «transformée»:

La transformation filmique présentera donc des différences par rapport au texte littéraire, liées à la matérialité spécifique de chaque médium, mais récupérables à travers une analyse de leur fonctionnement à l'intérieur du système textuel ou de leur récurrence thématique dans un texte particulier. ${ }^{16}$

Tout en insistant sur les influences tant du contexte sociohistorique que du marché, Jeanne-Marie Clerc note que limiter le débat à celui de la prééminence du textesource est assez restrictif. Lorsqu'un réalisateur s'empare d'une œuvre littéraire, il ne se sent pas lié de quelque manière que ce soit à elle. Il ne s'agit que d'une banque de données dont il dispose à sa guise. Pour Clerc,

[...] la transposition s'apparente à une création autonome dans la mesure où, même si elle emprunte certaines idées directrices à une ceuvre antérieure, elle conserve la liberté de les exprimer sous la forme qu'elle choisit. Resterait à déterminer si les contraintes propres à l'instrument d'expression ne pèsent pas sur ces choix, dans le cas de la caméra surtout. ${ }^{17}$

Décrite dans son livre Pour une théorie de l'adaptation filmique, l'approche de Patrick Cattrysse, dite "polysystémique", se base sur l'analogie avec les sciences de la traduction. Telle que menée, la démarche autorise quelques interrogations. Si Cattrysse s'émancipe de la recherche de fidélité, il demeure que l'analogie avec la traduction est en soi délicate puisque, même s'il ne semble pas l'admettre, il existe une nécessité théorique en traduction: l'interdiction de retrancher, ce qui implique un type singulier de fidélité.

Par l'étymologie même du terme, la traduction, comme l'adaptation, est proprement impossible, «infidèle», elle constitue un leurre royal et soulève de nombreux désagréments. C'est pourquoi certains auteurs (Milan Kundera par exemple) surveillent et approuvent même les traductions de leurs ouvrages. La recherche des équivalences, telle que suggérée à certains moments par l'auteur pour l'adaptation, ne constitue que des recours ultimes, même pour les cas des traductions ou des mises en scène. Bien plus: le traducteur ne s'attribue pas le loisir de "tailler» ou de "taillader» une œuvre, pour emprunter cette métaphore à Garcia, contrairement à une réécriture basée sur le principe de l'économie textuelle. C'est pourquoi le montage et l'ellipse sont essentiels tant dans les réécritures filmiques que dans les films faits à partir de scénarios originaux. Cattrysse laisse entrevoir aussi une équation entre le film et la langue, ce qui apparaît pour le moins improbable, comme l'a montré Christian Metz dans son ouvrage Langage et Cinéma. Il serait à cet égard intéressant de voir une traduction "polysystémique», par laquelle on retrancherait ou ajouterait des passages ou qui donnerait ce qu'a fait Benjamin-Jules Rosette de L'Aventure ambiguë: il ramène son film aux 17 premières pages du roman de Cheikh Hamidou Kane. Ne demeure-t-on pas en présence d'un texte gardant sa cohérence propre? Il est pourtant possible d'aborder la question sous un angle purement structural.

Se basant sur l'« Introduction à l'analyse structurale des récits» de Roland Barthes, Brian McFarlane distingue deux types d'opérations dans le déplacement: le transfert ("transfer») et l'adaptation proprement dite ("adaptation proper»). La première, le transfert, concerne la narrativité et la capacité à raconter. Depuis la Logique du récit de 
Claude Brémond, il s'agit du fait poétique le plus facilement exportable. Selon McFarlane, le transfert est plus aisé et ne nécessite pas de mutation majeure. Il porte sur l'histoire, l'intrigue, les différents personnages, les diverses fonctions et les sphères d'action barthésiennes. La deuxième opération, l'adaptation proprement dite, concerne par contre l'énonciation qui ne serait pas exportable et nécessiterait, selon McFarlane, un ajustement médiatique. Car la pluralité des codes au cinéma (verbal, visuel, auditif, gestuel) et la spatialité - qui different fondamentalement de la linéarité du roman -, ainsi que l'émergence des sons et les codes culturels sont des paramètres réglant la production des images industrielles; paramètres qui permettent de resituer autrement à l'écran les films d'origine littéraire. La primauté n'est plus au mot, mais à l'image et à ses codes. Cette étanchéité est plus marquée, voire consacrée, avec la récente théorie de la transécriture. On en arrive à se demander ce qui, dans ce qui survit au média au cours du processus de création, est véritablement «intermédial»: le code, la technologie, les langages ou l'acte poétique de création?

En effet, la séparation définitive des moyens d'expression pour justifier la différence textuelle est énoncée de façon rigoureuse, voire radicale, par André Gardies ${ }^{18}$. Opérant un important virage dans sa pensée, il estime que l'évaluation esthétique ne peut être considérée dans une relation comparative au texte-source. La raison évoquée est la différence de média ${ }^{19}$. Après Bluestone, Baby, Coreman, Clerc et McFarlane, la transécriture fait définitivement voler en éclats le mythe de la fidélité. La différence du texte d'arrivée est assurée par la différence du véhicule sémiotique qui le porte. Mais, pour emprunter à Bazin, une servitude succède en fait à une autre, une fidélité succède à une autre, presque innocemment. La transécriture accomplit ainsi une coupure épistémologique pour réinstaller la problématique dans la case départ, par une discrète inversion. André Gaudreault et Philippe Marion:

Trouver les formes de fidélité à l'esprit du média... Une telle fidélité serait probablement plus productive que la fameuse fidélité à l'auteur, ou encore, celle envers "le sujet" comme on dit. Toute adaptation qui se respecte se devrait alors d'organiser la violence faite à la fabula et au syuzhet de l'auvre de départ, parce que, d'une certaine manière, la nouvelle mise en syuzhet [...] relève non seulement de la "mise en sujet" d'une idée, mais aussi et surtout de sa mise en "sujétion». De son assujettissement au [...] média. ${ }^{20}$
On le voit, comme toutes les théories précédentes, celle de la transécriture généralisée "parie sur la différence», pour emprunter l'expression à Jeanne-Marie Clerc. Mais un problème ressurgit, car cette différence consubstantielle à tout déplacement semble uniquement légitimée par la nature du média cinématographique, lequel est investi du pouvoir autrefois attribué à la littérature: ici triomphe encore l'idéologie du média, l'essentialisme codique. En plus de continuer à chercher en quoi consiste exactement «l'esprit» d'un texte littéraire, il faudra aussi déterminer celui du média, en attendant sa «lettre». La répétition de la fidélité n'a pas l'air totalement innocente. Le déplacement sémiotique rejoint là ce déterminisme technologique qui a triomphé dans les années soixante avec cette formule de Marshall McLuhan: «En réalité et dans la pratique, le message, c'est le médium lui-même» ${ }^{21}$. Les formules nous semblent là comporter quelques mines (dans les deux sens) que la théorie des médias pourrait s'employer à déceler. Car si les changements ne se justifient que par le système sémiotique, que dirait-on alors des répétitions se déployant dans le même genre ou dans le même média? Autrement dit, où situer désormais, pour le cas du cinéma, le remake? Comment peut-on dorénavant, au vu de ce qui précède, élucider les écarts entre le Mutiny on the Bounty de Lewis Milestone (1935) et celui Frank Lloyd (1962), puisque nous demeurons dans le domaine du cinéma? Et les innombrables versions théâtrales, romanesques, poétiques et même chorégraphiques du même titre? Où situer désormais Notre-Dame de Paris qui triomphe dans les salles de spectacles?

Il semble important, à cet égard, de reconsidérer cette question de la reprise qui tient moins de la nature des codes expressifs que d'une poétique, celle de la répétition, principalement celle de la reprise. Il s'agit en fait d'une question de reprise, d'un dédoublement qui peut être infini. Sous le même se cache désormais un ou plusieurs autres. Le titre devient ainsi un signe doublé, triplé, en tout cas pluriel, quels que soient les registres ${ }^{22}$. La problématique semble donc moins celle des moyens sémiotiques que de la répétition. Bertrand Gervais, dans La Transécriture, le relève rapidement:

Car il y est question de double, de dédoublement, et même de doublure. Or qu'est-ce que l'adaptation sinon justement la création d'un double, la reprise d'un original [...]? Cette répétition repose sur l'argument qu'au-delà des mots et des 
images, qu'au-delà de leurs différences sémiotiques [...] un même travail d'imagination est accompli. 23

On situe dès lors toute l'importance de la réécriture. Non seulement elle implique, comme toutes les autres approches, une mutation liée à la reprise, mais elle se préserve aussi de toute extériorité, en ne fondant pas son régime de vérité sur un seul code ou un seul genre.

\section{Au-delà des codes et des genres:}

\section{réécriture et poétique de la répétition}

La fidélité, quel qu'en soit le degré, tient donc définitivement lieu de projet fictif. Elle fonde sa légitimité sur une conception restreinte de l'œuvre artistique, puis sur une capacité de transformation conférée par la puissance du code. Il importe pourtant de situer toute mutation non pas par rapport au code, mais par rapport à l'acte qui le fonde, à sa fréquence, à sa chronologie. Laquelle fait intervenir dans le procès poétique une autre signature, un autre sujet, une autre sensibilité qui approche le textesource en se définissant comme altérité - par laquelle s'esquisse un processus à part entière.

En effet, à des degrés divers, les théoriciens du déplacement sémiotique admettent que la réalisation filmique d'une œuvre littéraire est un acte de subjectivité, un fait de signature, même s'il implique toujours, comme le disent Jacques Derrida et Jean-François Lyotard, un «autre». La déception consécutive à la quête de la fidélité et des correspondances est le fait du lecteur ou du public qui, pour emprunter les termes à Christian Metz, voit ses fantasmes violentés et inaccomplis, dans la mesure où «il ne retrouve pas toujours son film, car ce qu'il a devant lui, avec le film véritable, c'est à présent le fantasme d'autrui, chose rarement sympathique» 24 . Ainsi, le véritable enjeu du déplacement sémiotique, comme celui de la réécriture, c'est aussi celui d'une substitution de fantasmes. Pour Jeanne-Marie Clerc ${ }^{25}$, il s'agit de rien moins q'une «aventure intérieure», dans la mesure où la réalisation d'un film est un cheminement que l'on porte en soi, et qui permet aussi au réalisateur de se "projeter" en cours de tournage. La fidélité est même ici déplacée vers ce réalisateur qui prend sur lui, en se substituant à l'auteur transposé, de reporter sur son ego les motivations de la nouvelle aventure artistique. François Baby, Brian McFarlane, Alain Garcia, Geofrey Wagner et André Gaudreault, à divers degrés, concèdent au réalisateur la liberté de façonner l'œuvre originale. Alain Garcia utilise à cet effet une métaphore heureuse, quoique prescriptive: il s'agit de tailler une ouvre comme un jardinier taille son buisson, et non de... «taillader». Il est donc question de rendre esthétique une économie interne et non de subir le diktat d'un modèle ou d'un code.

Toutes ces considérations déstabilisent complètement l'embarras de la fidélité en ce sens que l'œuvre littéraire n'est pas une donnée immuable. Elle ne comporte pas un sens, ni un discours vers lequel le cinéaste devrait diriger sa quête. Elle est simplement une banque de données comme le dit André Gardies: des données qui, même si elles ne sont pas en vrac, car elles constituent un ordre littéraire, sont réordonnées suivant une perspective nouvelle et descriptible. Le réalisateur choisit des éléments qu'il organise à sa convenance, en fonction de ses objectifs et, surtout, du public-cible et de la loi du marché - donnée cruciale dans le cinéma occidental. Lyotard l'écrit tout à propos: «Faire droit au différend, c'est instituer de nouveaux destinataires, de nouveaux destinateurs, de nouvelles significations, de nouveaux référents $[. ..]{ }^{26}$. Il peut, pour ces raisons, insister sur des éléments qui semblent secondaires dans le texte-source, ou consacrer entièrement son film à un seul aspect de l'œuvre romanesque comme le fait Benjamin Jules-Rosette dans sa version de L'Aventure ambiguë. Quel fondement sémiotique justifierait une accusation de charcutage ou de réduction? Peut-on se plaindre des nombreuses versions de Germinal?

Une telle option n'est jamais gratuite, tout comme ne l'est jamais un acte créateur. Luce Irigaray le disait à propos de la sexuation de la langue, "parler n'est jamais neutre»: réécrire encore moins. En insistant sur tel ou tel aspect de l'œuvre originale, en renonçant à tel ou tel autre, le film se constitue, dans la perspective idéologique ainsi que la décrit C. Kenneth Pellow en critique du roman. Loin de tout uniformisme sémantique ou interprétatif, le roman est une source infinie de significations. Comme le dit Umberto Eco, la réécriture est ainsi déterminée par une œuvre nécessairement ouverte:

[...] toute cuvre d'art, alors même qu'elle est forme achevée et "close» dans sa perfection d'organisme exactement calibré, est "ouverte" au moins en ce qu'elle peut être interprétée de différentes façons sans que son irréductible singularité en soit altérée. Jouir d'une ceuvre d'art revient à en donner une exécution, à la faire revivre dans une perspective originale. 27 
On l'a perçu, la reprise filmique des œuvres littéraires relève d'une esthétique de la réception. À la suite de Hans Robert Jauss, Jean-Pierre Esquenazi ${ }^{28}$ soutient, en parlant de l'adaptation, qu'elle est d'abord lecture, accueil d'une œuvre initiatrice, interaction entre texte et lecteur. Il ne s'agit nullement de vénérer la source comme un absolu, car le réalisateur produit une œuvre nouvelle, fût-ce dans des conditions sémiotiques différentes. Un tel travail de recréation s'effectue selon les mêmes principes poétiques, car il s'agit de doubler un texte d'un autre. Ce double estil, de ce fait, moins texte ou un texte moindre?

Certainement pas. Tout comme le texte tuteur, il est, au sens de Jacques Derrida, un "système de signification " 29 . L'enjeu est celui des modifications engendrées par la chronologie et, conséquemment, par la fréquence. Car en passant de la littérature au cinéma, on va d'une écriture à une autre - une écriture seconde suit une écriture première. Une écriture se greffe sur une autre, instaurant un écart différentiel entre l'antériorité et la postériorité. La doublure instaure une gemellité qui génère le paradoxe apparent de la déconstruction de l'un pour la construction de l'autre. Marie-Claire Ropars-Wuillemiers:

[...] le cinéma aux lieu et place de la littérature: mais en cela précisément le film appartient à l'écriture, qui devra se constituer sur la destitution de l'écriture originale. Le paradoxe du rapports'offre ici avec vigueur: à la fois écriture et lecture, répétition du même et invention de l'autre, l'ouvre filmique procède du désouvrement, puisqu'elle ne peut que se développer dans l'altération du texte devenu double - identique à soi et pourtant différent. Cette aptitude à la différence spécifie l'intervention du cinéma dans l'espace littéraire: soumettre tant l'ouvre à l'exigence d'un autre langage, qui l'affecte à son tour. La réécriture cinématographique détient ce pouvoir contraire de lire en écrivant, commenter en modifiant, dévoiler en masquant - et détruire sa propre originalité en réfutant l'unicité de l'origine. 30

Il apparaît à partir de ce qui précède que le concept de réécriture n'a été pensé jusqu'ici que dans le cadre des transmédiatisations, c'est-à-dire dans les cas impliquant un changement de mode d'expression. Si Marie-Claire RoparsWuillemiers admet le principe de la répétition comme fondement théorique de la différence et de l'altérité du texte d'arrivée, elle cède aussi, comme les tenants du déplacement sémiotique, à la tyrannie du code sémiotique. Or, ainsi que nous l'avons relevé plus haut, l'argument est moins celui du moyen que celui de la fréquence et même de la chronologie. À la prééminence de la littérature qui fait du fantasme de la fidélité un opérateur critique, succède la prééminence du cinéma. Après cette primauté mythique des origines dont parle Mircea Eliade, ressurgissent l'absolu et l'essentialisme du code tel que l'établissait McLuhan. Ce qui est contraire à une poétique de la réécriture:

La perspective critique de la réécriture refuse tout dogmatisme [y compris donc celui du code], et par avance réfute celui qui naîtrait de lui, puisqu'aussi bien elle montre que le texte littéraire, depuis l'identique, invente l'autre, depuis la répétition, la variation, et dans les deux sens. ${ }^{31}$

On marque là une étape importante dans la réflexion théorique: la réversibilité supplante l'unicité de l'origine. Dans un court article, «L'autre du même», Gérard Genette indiquait déjà qu'on ne peut répéter sans varier, ni varier sans répéter. Ces prémisses n'orientent pas, n’ordonnent pas l'acte sémiotique, ne conferent pas une direction fixe. Le geste de sémiotisation pourrait bien aussi aller de la littérature vers le cinéma, la bande dessinée ou le ballet, du roman vers le théâtre, de la nouvelle vers le film. Bref, plusieurs parcours sont possibles et réalisables. En dehors de Marie-Claire Ropars-Wuillemiers, seule Jeanne-Marie Clerc parle assez rapidement du concept de réécriture. Et pour elle, le phénomène concerne les auteurs qui portent eux-mêmes leurs œuvres à l'écran. Dans tous les cas de figure, d'un genre à un autre, d'un mode d'expression à un autre, d'une époque à une autre, une œuvre tutrice subit un démembrement qui permet d'assurer le remembrement d'une ouvre seconde qui la double, la dédouble, la redouble, la dépasse par sa différence. Une telle approche libère définitivement du paradoxe de la fidélité qui est l'un des concepts les plus incertains et les moins défendables, mais le plus tenace, qui ont émaillé la poétique des différents mouvements textuels. Elle affranchit aussi la recherche sémiologique d'une régence remaniée par les théoriciens de la transécriture: celle du code sémiotique et de l'idéologie du média qui, seuls, justifieraient toute innovation esthétique.

Car ce qu'on oublie, c'est que le patrimoine littéraire universel regorge de versions multiples du même titre. Des Fables d'CEsope à celles de La Fontaine, les nombreux textes titrés Don Quichotte, les multiples aventures d'CEdipe, de 
Chaka, de Robinson Crusoé ou d'Antigone, les parcours initiatiques d'Ulysse (dans L'Odyssée d'Homère, Ulysse de James Joyce, Molloy de Samuel Beckett), tous ces exemples permettent de mesurer l'ampleur des perspectives de la réécriture. Pourtant, aucun de ces ouvrages ne semble générer de débat ou de remise en question esthétique majeure, bien que tous détruisent une origine pour en constituer une autre. Prenant sans doute la mesure du phénomène et de la suspicion qui afflige le terme "adaptation", trop réducteur pour lui, Milan Kundera a appelé sa version de Jacques le fataliste une "variation» 32 . Ainsi, il ne se cache pas derrière le roman pour en reconstituer un sens improbable. On ne parle donc pas, dans ces cas, de "trahison" ou de «fidélité». Pourquoi pas? La raison informulée est le partage du code et éventuellement du genre. On digère et soutient les différences de formes et de contenus pour la simple raison qu'elles sont convoyées par le même langage.

Il faut pourtant admettre que, de l'avis de plusieurs théoriciens (Gaudreault et Ropars-Wuillemiers après Kundera), le terme «adaptation" pose problème. Le fantôme du texte fondateur vient piper toute perspective critique car il implique une esthétique de la représentation, de la restitution. Réécriture peut sauver d'un tel embarras. Car cette esthétique institue un acte odipien émancipateur par l'instauration effective de l'autonomie, de l'altérité, laquelle se fonde sur la répétition d'un acte de création. Le meurtre du texte parent est une nécessité poétique, car il permet au sujet de se constituer sur lui, par un effacement régénérateur et transparent (Maurice Blanchot), contre lui. Même les dictionnaires concèdent à la réécriture le geste (et la geste) d'une écriture nécessairement différentielle: réécrire c'est écrire ou rédiger de nouveau ce qui est déjà écrit, en modifiant au lieu de copier à l'identique. Or cela est connu, la copie n'est jamais conforme. C'est pourquoi il faut la certifier. La certification tenant lieu d'aveu d'impuissance face à l'altérité fondamentale du même qui n'est jamais tout à fait même: toujours altéré, affecté par le mouvement, l'espace et le temps. Le préfixe de la réécriture est à ce titre le signe fondateur qui opère la rupture de l'identique en suscitant, par le retour (éternel) du même, un écart différentiel lié à la répétition. C'est du reste l'une des thèses de Gilles Deleuze:

Ce sont toujours les différences qui se ressemblent, qui sont analogues, opposées ou identiques: la différence est derrière toute chose, mais derrière la différence, il n'y a rien. [...] C'est pourquoi l'éternel retour ne surgit pas en second, ou ne vient pas après, mais est déjà présent dans toute métamorphose, contemporain de ce qu'il fait revenir [...] La répétition s'oppose à la représentation, le préfixe a changé de sens car dans un cas, la différence se dit seulement par rapport à l'identique, mais dans l'autre cas, c'est l'univoque qui se dit par rapport au différent. La répétition, c'est l'être informel de toutes les différences, la puissance informelle du fond qui porte chaque chose à cette "forme» où sa représentation se défait. 33

Du point de vue théorique, Maurice Blanchot aborde très rapidement la question dans Le Pas au-delà. Dans une perspective qui est proche de l'intertextualité généralisée, telle que la développera plus tard Roland Barthes, il fait de la question de la réécriture l'acte sémiotique lié à toute création ${ }^{34}$. L'écriture est pour lui forcément réécriture:

Écrire, c'est peut-être non-écrire en écrivant - effacer (en écrivant par-dessus) ce qui n'est pas encore écrit et que la réécriture non seulement recouvre, mais restaure obliquement en la recouvrant, en obligeant à penser qu'il y avait quelque chose d'antérieur, une première version (détour) ou, pis, un texte d'origine et par là nous engageant dans le processus de lillusion du déchiffrement infini. 35

Du point de vue littéraire, le groupe de recherche Grelis a consacré un numéro spécial à la question: La Réécriture du texte littéraire (Semen $\mathrm{n}^{\circ} 3$, 1987). La problématique $\mathrm{y}$ est abordée de manière frontale, à partir de divers exemples littéraires. Le $\mathrm{n}^{\circ} 30$ de la revue Littératures (1978) est aussi partiellement consacré à cette problématique. Au cinéma, du fait des remakes et d'autres réécritures, il existe de nombreuses variations sur un même titre. L'origine est destituée dans ces cas par des textes qui jouent le jeu de leur autoorigination: ils prennent naissance dans l'effacement d'une autre origine. Telle se présente la problématique de la réécriture qui n'a pas uniquement l'avantage de consolider la différence comme nécessité. Elle décloisonne les genres littéraires et les médias en faisant de la répétition le lieu d'une nouvelle élaboration textuelle et d'une altérité fondée sur la reprise, la destitution/constitution d'une identité. Dans un tel contexte, la signature et le sens du mouvement poétique permettent de la qualifier. On pourrait ainsi éviter quelques barbarismes ou de simples aménagements terminologiques, et déterminer en quelque sorte le sens de l'opération sémiotique opérée par un signataire. On parlera alors 
de réécriture filmique (le cas le plus répandu), littéraire (Ousmane Sembène avec Guelwaar), romanesque ou théâtrale (Kundera et son Jacques), etc.

Il faut dire que la théorie ne considérait jusqu'ici la poétique de la réécriture qu'à sens unique: de la littérature vers le cinéma ou, dans le cadre du n 03 de la revue Semen, d'un texte littéraire vers un autre. Or, si l'on admet avec Christian Metz que toute écriture est une activité de composition, comme toute activité artistique en général, on comprend dès lors, ainsi que l'avait indiqué Derrida depuis longtemps, que l'écriture en tant que phénomène esthétique est extensible à tout système de communication. De ce fait, elle survit aux médias et aux genres. Cette caractéristique s'applique dès lors à sa répétition, la réécriture. Ousmane Sembène a bien tiré du scénario de Guelwaar un roman. Pour son cas, comme pour celui de Bassek Ba Khobio, de Jean Giono, d'André Malraux et, dans une certaine mesure, celui d'Abdoulaye Mamani qui a participé avec Med Hondo à la réalisation de Sarraounia, on parlera non pas de réécriture au sens que suggère Jeanne-Marie Clerc, mais d'autoréécriture. Cela veut dire que le réalisateur du film est aussi l'auteur du roman, qu'il «s'origine», qu'il a deux origines, qu'il est dans l'entre-deux-origines. Il n'y en a pas une plus vraie que l'autre. Ces exemples de double identité, par laquelle le même titre désigne/appelle plusieurs textes, disqualifient encore davantage toute problématique de fidélité ou de trahison. Il faudra dès lors trouver d'autres critères, nécessairement esthétiques et idéologiques, pour justifier des variations entre les versions littéraires et filmiques de Sango Malo, Sarraounia, Hamlet, Les Misérables et de Guelwaar par exemple. Ces cas de double identité règlent en outre certaines interrogations quant à la question de la signature et de l'auteur: ce qu'un texte devient d'un code à un autre n'est certainement pas le fruit d'un hasard. Dans les autres cas, les plus nombreux, où l'auteur de l'œuvre cinématographique s'approprie le texte d'autrui, moyennant des droits, on parlera, s'il importe d'être plus précis, d'hétéro-réécriture. Ainsi Jacques Champreux et Benjamin Jules-Rosette s'emparent de L'Aventure ambiguë de Cheikh Hamidou Kane et Laurent Chevalier de L'Enfant noir de Camara Laye. Ou de Kenneth Branagh qui a produit récemment sa version de Hamlet (1996). On dira la même chose des réécritures filmiques telles que l'histoire du cinéma en compte. Ainsi apparait, dans ses grandes lignes, ce qui nous semble constituer la problématique fondamentale de toute reprise.
Cette étude a tenté de situer la réécriture dans les divers registres théoriques existants. On l'a vu, les derniers développements consolident une simple permutation quant aux pôles du pouvoir: de celui de la littérature toute-puissante, on arrive à celui du cinéma, non moins puissant. La poétique se trouve ainsi prise dans une espèce de double bind dont il serait temps de se déprendre. Qu'il s'agisse du passage de la littérature vers le cinéma ou, au contraire, du roman vers le théâtre ou la bande dessinée, ce qui est mis en œuvre est un ensemble de modalités dans les reprises. En ce sens, la réécriture marque sa distance avec les tenants du déplacement sémiotique. Ce qui engage la différence, c'est peut-être aussi le code expressif, mais c'est d'abord la fréquence de l'acte poétique. L'écriture est à l'œuvre dans tous les systèmes de communication, disent Derrida et Metz, et toute répétition implique altération, laquelle est toujours déjà effective par l'usure du temps et de l'espace. Il n'existe pas d'essence. Le même n'est jamais tout à fait le même, ni l'autre tout à fait autre. C'est pourquoi la gemellité qu'introduit la réécriture impose de réfléchir sur le statut du texte dérivé.

\section{Notes}

1. A. Bazin, 1959: 18

2. A. Gaudreault et T. Groensteen, 1998.

3. A. Bazin, 1959: 21.

4. Ibid.

5. A. Garcia, 1990: 15.

6. G. D. Philips, $1980: 158$. Je souligne.

7. C. Orr, 1984: 72. Je souligne.

8. A. Garcia, 1990: 202-203.

9. F. Baby, $1980: 12$.

10. Ibid.

11. G. Wagner, $1975: 222$

12. Ibid., p. 224.

13. Ibid., p. 226. Je souligne.

14. M.-C. Ropars-Wuillemiers, $1990: 160$

15. G. Bluestone, 1966: 5 .

16. L. Coreman, 1990: 33.

17. J.-M. Clerc, 1985: 31.

18. A. Gardies, 1998: 80.

19. Il écrit à peu près le contraire ailleurs en admettant qu'il est possible de discuter de la question esthétique "à condition de procéder à une comparaison non sur la base des équivalences, mais en procédant par comparaison et contraste», A. Gardies, 1993: 7. Je souligne.

20. "Transécriture et médiatique narrative», op. cit., p. 52. Je souligne. 21. Pour comprendre les médias, 1968, p. 25. Je souligne.

22. Un exemple: à ce jour, exactement 36 versions filmiques pour Les 
Misérables de Victor Hugo. Les différences se situent-elles au niveau technologique ou poétique?

23. B. Gervais, 1998: 108-109. Je souligne

24. C. Metz, 1977: 137. Souligné par l’auteur.

25. J.-M. Clerc, 1985: 22.

26. J.-F. Lyotard, 1983: 29.

27. U. Eco, 1967: 17, souligné par l'auteur.

28. J.-P. Esquenazi, 1996: 37-44.

29. J. Derrida, 1967: 135 .

30. M.-C. Ropars-Wuillemiers, 1990: 170.

31. M. Domino, 1987: 37. Je souligne.

32. Lire M. Kundera, 1981: 7-20.

33. G. Deleuze, 1968: 80. Souligné par l'auteur.

34. Ne faudrait-il pas, à cet effet, comme les marxistes, parler davantage de production, plutôt que de création dans le cas de la réécriture? 35. M. Blanchot, 1971: 67.

\section{RÉFÉRENCES BIBLIOGRAPHIQUES}

Films

Aventure ambiguë (L'), réalisation Jacques Champreux, 1992; réalisation Benjamin Jules-Rosette, 1991.

Enfant noir (L'), réalisation Laurent Chevalier, 1993.

Guelwaar, réalisation Sembène Ousmane, 1992.

Hamlet, réalisation Celestino Coronado, 1976; réalisation Kenneth Branagh, 1996.

Misérables (Les), réalisation J. Stuart Blackton, 1909; réalisation Frank Lloyd, 1918; réalisation Raymond Bernard, 1933 réalisation Mara Mattuschka, 1987; réalisation Claude Lelouch, 1995.

Mutiny on the Bounty, réalisation Frank Lloyd, 1935; réalisation Lewis Milestone, 1962.

Sarraounia, réalisation Med Hondo, 1986.

Sango Malo. Le Maître du canton, réalisation Bassek Ba Kobhio, 1991.

Xala, réalisation Sembène Ousmane, 1974.

Ouvrages théoriques et analyses diverses

BABY, F. [1980] : «Du littéraire au cinématographique: une problématique de l'adaptation", Études Littéraires, avril, 11-29.

BAZIN, A. [1959]: Qu'est-ce que le cinéma? II: Le cinéma et les autres arts, Paris, Le Cerf.

Blanchot, M. [1971] : Le Pas au-delà, Paris, Gallimard.

Bluestone, G. [1966]: Novels into Films, Berkeley and Los Angeles,
University of California Press.

CATtRYsse, P. [1992]: Pour une théorie de l'adaptation filmique. Le film noir américain, Berne, Peter Lang.

CleRC, J.-M. [1985] : Écrivains et cinéma. Des mots aux images, des images aux mots, Metz, Presses Universitaires de Metz.

COREMAN, L. [1990] : La Transformation filmique. Du Contesto à Cadaveri Eccellenti, Berne, Peter Lang.

DeleuZe, G. [1968] : Différence et Répétition, Paris, P.U.F.

DERRIDA, J. [1967] : De la grammatologie, Paris, Minuit.

DOMINO, M. [1987] : «La réécriture du texte littéraire. Mythe et réécriture», dans Grelis 3, 13-37.

ECO, U. [1967] : L'CEuvre ouverte, Paris, Seuil.

ESQUENAZI, J.-P. [1996] : «De l'adaptation à l'ingestion. ", Cinémaction 76.

GARCIA, A. [1990] : L'Adaptation du roman au film, Paris, I.F, Diffusion Dujaric.

GARDIES, A. [1993] Le Récit filmique, Paris, Hachette;

[1998] «Le narrateur sonne toujours deux fois», dans A. Gaudreault et T. Groensteen, 65-80.

GaUdreault, A. et P. MARION [1998] : "Transécriture et médiatique narrative. L'enjeu de l'intermédialité", dans A. Gaudreault et T. Groensteen, 31-54.

GAUdreault, A. et T. GROENSTEEN (sous la dir.) [1998] : La Transécriture. Pour une théorie de l'adaptation, Québec/Angoulème, Nota Bene Centre/ National de la Bande Dessinée.

GenetTe, G. [1985] : «L'autre du même», Corps Écrit 15, Répétition et Variation, 11-15.

GERVAIS, B. [1998]: «Torsions, distorsions ou comment adapter une méprise de lecture », dans A. Gaudreault et T. Groensteen, 107-130.

GRELIS [1987] : La Réécriture du texte littéraire, dans Semen no 3, Paris, Les Belles Lettres.

Jauss, H. R. [1978] : Pour une esthétique de la réception, Paris, Gallimard. KUNDERA, M.[ 1981]: «Introduction à une variation", Jacques et son maître. Hommage à Denis Diderot en trois actes, Paris, Gallimard, 7-20.

LITTÉRATURES n ${ }^{\circ} 30$ [1978] : Motifs, Transferts, Réécriture, Paris, Larousse, mai.

LYOTARD, J.-F. [1983] : Le Différend, Paris, Minuit.

MCFarlane, B. [1996] : Novel to Film. An Introduction to the Theory of Adaptation, Oxford, Calendon Press.

McLuhan, M. : [1968] Pour Comprendre les médias, Paris, Seuil.

METZ, C. [1971] : Langage et Cinéma, Paris, Larousse;

[1977] : Le Signifiant imaginaire, Paris, U.G.E.

ORR, C. [1984] : «The Discourse on Adaptation ", Wide Angle, 6/2, 72-76.

PELLOW, C. K. [1994]: Films as Critiques of Novels. Transformational Criticism, Lewingston, The Edwin Mellen Press.

PHILIPS, G. D. [1980] : Hemingway and Film, New York, Ungar Publishing. ROPARS-WuILlEMIERS, M.-C. [1990] : Écraniques. Le film du texte, Lille, Presses Universitaires de Lille.

WaGner, G. [1975]: The Novel and the Cinema, Rutherford, N.J., Fairleigh Dickinson University Press. 